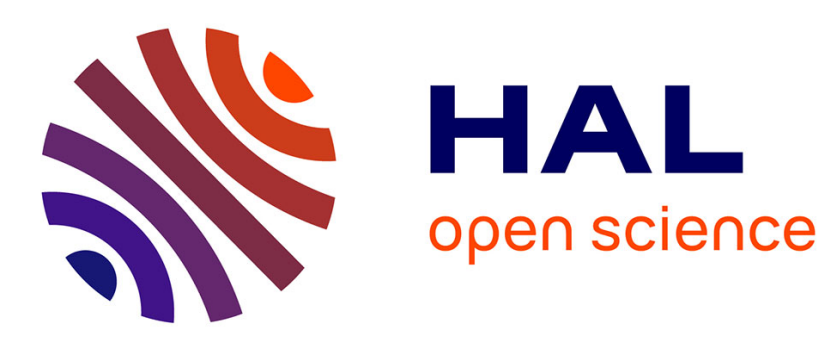

\title{
Passive guided waves measurements using fiber Bragg gratings sensors
}

Tom Druet, Bastien Chapuis, Jules Manfred, Guillaume Laffont, Emmanuel Moulin

\section{- To cite this version:}

Tom Druet, Bastien Chapuis, Jules Manfred, Guillaume Laffont, Emmanuel Moulin. Passive guided waves measurements using fiber Bragg gratings sensors. Journal of the Acoustical Society of America, 2018, 144 (3), pp.1198-1202. 10.1121/1.5054015 . hal-03183485

\section{HAL Id: hal-03183485 \\ https://hal.science/hal-03183485}

Submitted on 6 Apr 2021

HAL is a multi-disciplinary open access archive for the deposit and dissemination of scientific research documents, whether they are published or not. The documents may come from teaching and research institutions in France or abroad, or from public or private research centers.
L'archive ouverte pluridisciplinaire HAL, est destinée au dépôt et à la diffusion de documents scientifiques de niveau recherche, publiés ou non, émanant des établissements d'enseignement et de recherche français ou étrangers, des laboratoires publics ou privés. 


\title{
Passive guided waves measurements using fiber Bragg gratings sensors
}

\author{
Tom Druet, ${ }^{1}$ Bastien Chapuis, ${ }^{1}$ Manfred Jules, ${ }^{1}$ Guillaume Laffont, ${ }^{1}$ and Emmanuel Moulin ${ }^{2}$ \\ ${ }^{1)}$ CEA, LIST, F-91191 Gif-sur-Yvette, France ${ }^{\mathrm{a}}$ \\ ${ }^{2}$ IEMN UMR CNRS 8520, Université de Valenciennes et du Hainaut-Cambrésis, F-59313 Valenci- \\ ennes cedex 9, France
}

Guided elastic waves are often studied as an effective solution for Structural Health Monitoring (SHM) systems of plate-like structures thanks to their capabilities to propagate on large distances. In typical applications such as monitoring delaminations in aircrafts fuselage, a network of piezoelectric transducers (PZT) is used to emit and receive such waves in the structure. Fiber Bragg grating (FBG) sensors on optical fibers are a promising alternative to PZT for guided waves measurements in practical applications due to their dense multiplexing capabilities and robustness with respect to the environment.

However, FBG sensors cannot emit waves unlike conventional PZT transducers.

It is demonstrated here that FBG sensors can be used in combination with a passive diffuse noise cross-correlation technique in order to extract the coherent guided waves propagating between two sensors. This could lead to a system using only FBG sensors in the near future. The reconstructed signals can then be analyzed with usual guided waves algorithms, like in active SHM systems, keeping all the advantages of this kind of monitoring in terms of fine diagnosis.

The experimental demonstration shown in this paper is performed at ultrasonic frequencies $(20-100 \mathrm{kHz})$ typically used in guided waves based SHM systems showing the potential of the approach.

\section{INTRODUCTION}

Structural Health Monitoring (SHM) consists in acquiring and analyzing data from sensors embedded in a structure in order to evaluate its health (SAE International , 2013). A physical way to interrogate large and thin structures such as aircraft fuselages, pipes or ship hulls can be offered by guided elastic waves emitted and received by a network of PZT (Yu , 2008).

In the past years it has been shown that it is possible to use ambient structure-borne elastic noise (due to engine vibrations or aero-acoustic turbulences for example) instead of emitting actively the waves in the structure (Sabra , 2007). This technique, called ambient noise cross-correlation or passive technique, is based on the retrieval of the Green's function of the medium between two sensors from the correlation of the ambient noise (diffuse field) measured simultaneously by these two sensors (Lobkis , 2001). The link between the correlation and the Green's function has been verified experimentally and with simulations in different domains: ultrasound (Lobkis , 2001; Weaver , 2001; Derode , 2003'a; Larose , 2004), electromagnetism (Davy , 2016), structures vibration and/or SHM (Sabra , 2007, 2008; Chehami , 2014; Larose , 2007' 2009; Duroux , 2010' a; Sabra , 2011), ocean

a) tom.druet@cea.fr acoustic (Roux , 2004; Sabra , 2005a; Zang , 2015), helioseismology (Duvall , 1993; Gizon , 2004), medical (Sabra , 2007a; ) and seismology (Campillo , 2003; Shapiro , 2005, 2004; Sabra , 2005; Snieder, 2010; Zeng , 2017).

The passive technique has several advantages in the context of SHM (Sabra , 2008; Chehami , 2014). First, since it is not necessary to inject energy into the structure for wave emission, the power consumption of the SHM system can be significantly reduced. This should be of crucial importance for embedded systems with limited or no access to power sources like aircraft or underwater monitoring systems. Then, the complexity of both the electrical cabling and the embedded electronics is reduced, since no emission circuitry is necessary.

Another interesting possibility offered by this passive technique is to design a guided wave based SHM system with minimal intrusiveness thanks to the use of fiber Bragg grating (FBG) sensors on optical fibers. Indeed, FBG sensors have already been used as receivers of guided waves for SHM systems, but only in combination with PZT used to emit these waves (Betz , 2003; Takeda , 2005; Sotoudeh , 2014).

The use of optical fibers in combination with noise cross correlation has recently been studied in the literature with a technology called "distributed acoustic" sensing (DAS) (Zeng , 2017). DAS systems are based on Rayleigh backscattering caused by randomly distributed scattering sites along any optical fiber, coming either 
from non-uniform refractive index change along the optical fibers core or from tiny dimensional changes of the cores radius. DAS system launch coherent laser pulse into the optical fiber under test which is used to probe the scattering sits distributed all along the fiber. When returning back to the measurement system, the coherent backscattered light is mixed to a reference signal and the resulting interference signal is analyzed to retrieve the measurand of interest, usually dynamic strain induced into the optical fiber by acoustic signal impinging on the sensing cable. The spatial resolution is mainly determined by the pulse temporal width. Coherent laser pulses as short as $10 \mathrm{~ns}$ can be achieved using for instance an electro-optic modulator. In that case, a metric resolution along the fiber is obtained, making this system inapplicable for typical configurations of guided waves based SHM systems at ultrasonic frequencies (20-500 kHz). Indeed, these frequencies allow a good compromise between long propagation and good sensitivity to defect detection.

FBG are sensors of much smaller size (a few millimeters) adapted to sense the wavelengths of interest in a SHM context. The combination described in this paper of ambient noise cross-correlation and FBG sensors allows to determine the pitch-catch response (the response of the structure between two sensors for a given solicitation) between two FBG sensors as if one was used as an emitter. This offers all the advantages of purely optical fiber sensing (dense sensor multiplexing, immunity to and no generation of Electro-Magnetic Interferences, applicability in harsh explosive, high temperature, cryogenic, wetted, radiative-environments, no need of additional wiring) with the fine diagnosis offered by guided elastic waves (detection, location and sizing of defects, full structure coverage).

This article is aimed at demonstrating that the passive technique applied to FBG measurements can retrieve the information contained in active signals (the ones used in SHM diagnosis algorithms, for instance time-offlight (TOF) or full pitch-catch waveform). After a presentation of the methods and experimental setup used, a first experimental demonstration of passive measurements with FBG sensors is shown. Comparisons between active and passive signals are also described.

Note that the term active signal refers to the guided wave field measured at point $B$ after propagation from an emission placed at point $A$, whereas passive signal refers to the reconstructed guided wave field at point $B$ from the cross-correlation of the diffuse elastic noise measured simultaneously in $A$ and $B$.

\section{METHODS}

\section{A. Data processing}

(Weaver , 2001) demonstrated that the crosscorrelation (see Eq. 1) of diffuse ultrasonic noise yields the elastic Green's function of the medium $G$. The crosscorrelation of a wave field $\varphi$ sensed by two receivers at points $\boldsymbol{A}$ and $\boldsymbol{B}$ reads:

$$
C_{\boldsymbol{A} \boldsymbol{B}}(t)=\frac{1}{T} \int_{0}^{T} \varphi(\boldsymbol{A}, \tau) \varphi(\boldsymbol{B}, t+\tau) \mathrm{d} \tau .
$$

(Lobkis , 2001) showed that the time derivative of the cross-correlation (see Eq. 2) contains the exact Green's function of the medium, as if sources were placed at points $\boldsymbol{A}$ and $\boldsymbol{B}$ :

$$
\frac{\partial}{\partial t} C_{\boldsymbol{A B}}(t) \propto G^{+}(\boldsymbol{A}, \boldsymbol{B}, t)-G^{-}(\boldsymbol{B}, \boldsymbol{A}, t),
$$

with $G^{-}(\boldsymbol{B}, \boldsymbol{A}, t)=G^{+}(\boldsymbol{A}, \boldsymbol{B},-t)$ and $G^{+}$being the causal Green's function and $G^{-}$the anticausal one (time reversed). (Lobkis , 2001) demonstrated this relation for a bounded domain without attenuation, where the modes of the structure form a discrete set. More specifically, it is assumed that each mode of the structure is excited by the noise with the same energy whereas its phase is random (equipartition hypothesis). It should be noted that the time derivative in Eq. 2 depends on the considered Green's function. This is true if the Green's function links an impulsive force (the source excitation), to a displacement (the measurement), which will be the case of the experiment presented in this paper.

Active signals $s_{\boldsymbol{A} \boldsymbol{B}}$ are obtained by emission of a toneburst $E$ from a PZT placed in $\boldsymbol{A}$ and measurement of the guided waves field, after propagation, with an FBG sensor placed in $\boldsymbol{B}$. In our experiments, $E$ was defined as:

$$
\begin{cases}E(t)=\sin \left(2 \pi f_{0} t\right)\left(\frac{1}{2}-\frac{1}{2} \cos \left(2 \pi \frac{f_{0}}{n_{\mathrm{cy}}} t\right)\right) & \forall t \in\left[0, \frac{n_{\mathrm{cy}}}{f_{0}}\right], \\ E(t)=0 & \forall t \in \mathbb{R} \backslash\left[0, \frac{n_{\mathrm{cy}}}{f_{0}}\right],\end{cases}
$$

where $f_{0}$ is the central frequency and $n_{\mathrm{cy}}$ the number of cycles.

Passive signals $s_{\boldsymbol{A} \boldsymbol{B}}^{\mathrm{P}}$ are obtained following the subsequent procedure:

- simultaneous measurement of $\varphi_{\boldsymbol{A}}$ and $\varphi_{\boldsymbol{B}}$ using two FBG sensors or one FBG sensor and one PZT ;

- computation of the cross-correlation $C_{\boldsymbol{A} \boldsymbol{B}}$ of $\varphi_{\boldsymbol{A}}$ and $\varphi_{B}$ according to Eq. 1, which gives a broadband estimation of the Green's function (passive response);

- numerical filtering of the passive response.

The numerical filtering step depends on the application. Indeed, passive signals are generally broadband, and difficult to interpret directly. In order to compare them with theoretical TOF of propagating guided modes (see Fig. 3), a band-pass filter is applied here around a specific central frequency. In the case of a comparison with active signals, the following filtering is performed in order to guarantee the same spectral content and hence obtain meaningful comparisons:

$$
\begin{cases}\hat{s}_{\boldsymbol{A} \boldsymbol{B}}^{\mathrm{P}}(\omega)=\frac{\hat{C}_{\boldsymbol{A B}}(\omega)}{\left|\hat{C}_{\boldsymbol{A B}}(\omega)\right|}\left|\hat{s}_{\boldsymbol{A} \boldsymbol{B}}(\omega)\right| & \forall \omega \in \Omega_{\epsilon}, \\ \hat{s}_{\boldsymbol{A} \boldsymbol{B}}^{\mathrm{P}}(\omega)=\hat{C}_{\boldsymbol{A B}}(\omega) & \forall \omega \in \mathbb{R} \backslash \Omega_{\epsilon},\end{cases}
$$


where the hat refers to the Fourier transform of a variable and the set $\Omega_{\epsilon}$ reads $\Omega_{\epsilon}=$ $\left\{\omega \in \mathbb{R}|| \hat{C}_{\boldsymbol{A} \boldsymbol{B}}(\omega)\left|>\epsilon \max _{\omega}\right| \hat{C}_{\boldsymbol{A} \boldsymbol{B}}(\omega) \mid\right\}$, with $\epsilon$ a threshold taken as $\epsilon=0.01$ here. It should be noted that Eq. 4 ensures the same power spectral density between active and passive signals but leaves the phase of the cross-correlation unchanged.

\section{B. Experimental setup}

The experimental results presented in this paper have been obtained on a $2 \mathrm{~mm}$ thick, $1.25 \mathrm{~m}$ long and $1 \mathrm{~m}$ wide aluminum plate. The ambient noise has been generated in the plate by manually spraying compressed air randomly on its surface (Larose , 2007). The distance between the nozzle and the plate was of approximately $5 \mathrm{~cm}$. For the passive measurements, the acquisition time was $10 \mathrm{~s}$ (thus $10 \mathrm{~s}$ of noise where generated and post-processed) sampled at $2.5 \mathrm{MHz}$. For the active measurements, the acquisition time was $2 \mathrm{~ms}$.

Two FBG sensors A and B have been glued at the surface of the aluminum plate using a two component heat-curable epoxy adhesive. Both FBG sensors have a length of $2 \mathrm{~mm}$ and were photowritten in a polyimidecoated germanosilicate single-mode optical fiber. The optical fiber has been previously loaded with hydrogen at 200 bars during two weeks to increase its photosensitivity. Each FBG was interrogated by a separate tunable laser source locked to the low-wavelength side turning point of the Bragg spectral resonance. The FBG sensors have been inscribed at CEA List photowriting facilities using a continuous-wave frequency-doubled argon ion laser emitting an optical power of $100 \mathrm{~mW}$ at $244 \mathrm{~nm}$ and using a Lloyd mirror interferometer. The FBG sensors have a full-width-at-half-maximum of $800 \mathrm{pm}$. They are used as edge filters to convert any Bragg peak spectral oscillation induced by the elastic ambient noise into a modulation of the tunable laser optical power reflected by the gratings. This optical signal is then detected using Indium Gallium Arsenide (InGaAs) photodetectors, digitalized by an oscilloscope and stored for further post-processing.

\section{RESULTS}

In this section we first describe passive measurements between two FBG sensors and then show practical comparisons between active and passive signals using an FBG sensor and a PZT.

\section{A. Cross-correlation of FBG sensors' measurements}

For the first experiment presented here, sensors used were two FBG; hence, no comparison with active signals are shown since FBG sensors cannot be used as emitter.

Two FBG sensors spaced $40 \mathrm{~cm}$ apart from each other were used to measure the ambient noise. It is well know that FBG sensors have a directional measurement behavior but this is not the purpose of this paper. That is why it has been decided to align the sensors. The recorded noise was then cross-correlated to get the broadband passive signal of Fig. 1.

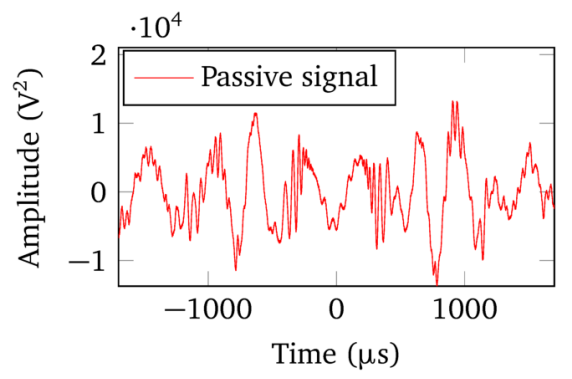

FIG. 1. (Color online) Cross-correlation between two FBG sensors noise measurements.

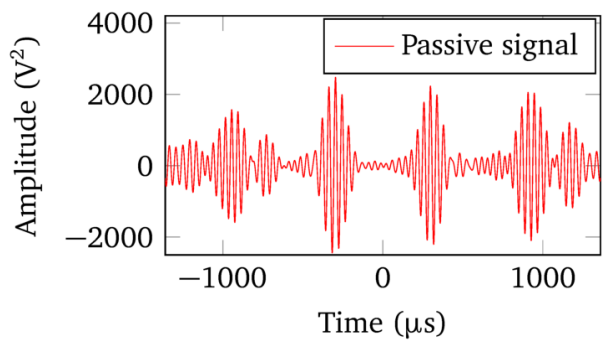

FIG. 2. (Color online) Signal of Fig. 1 which has been filtered (central frequency of $25 \mathrm{kHz}$ and bandwidth of $12.5 \mathrm{kHz}$ ).

On this figure it is already possible to assess the passive reconstruction quality by looking on its symmetry but this qualitative criterion is not sufficient. It is better to assess reconstruction's quality with a quantitative criterion and, for that, the theoretical TOF is used. First, the very wide band passive signal of Fig. 1 is filtered by a numerical band-pass filter with a given central frequency. As can be seen on Fig. 2 in the special case of a central frequency of $25 \mathrm{kHz}$, the wave packets are clearly separated, which enables us to analyze the signal in terms of TOF. By applying this band-pass filter for various central frequencies and taking the enveloppe of the signal, the TOF of a given mode corresponds to the time at which the amplitude of the corresponding packet is maximum. The squared signal envelopes for different central frequencies and the theoretical TOF of the aluminum plate $\mathrm{A}_{0}$ mode (fundamental antisymmetric Lamb mode) at such frequencies are plotted on Fig. 3. The time corresponding to the maximum of the envelope matches reasonably the theoretical TOF. The dispersion properties of $\mathrm{A}_{0}$ mode at this low frequency $\times$ thickness product are also visible on this figure. Indeed, when the frequency increases, one can see on Fig. 3 that the wave packets propagate faster. These results confirm the excellent passive reconstruction (in terms of signal to noise ratio) for this configuration. 


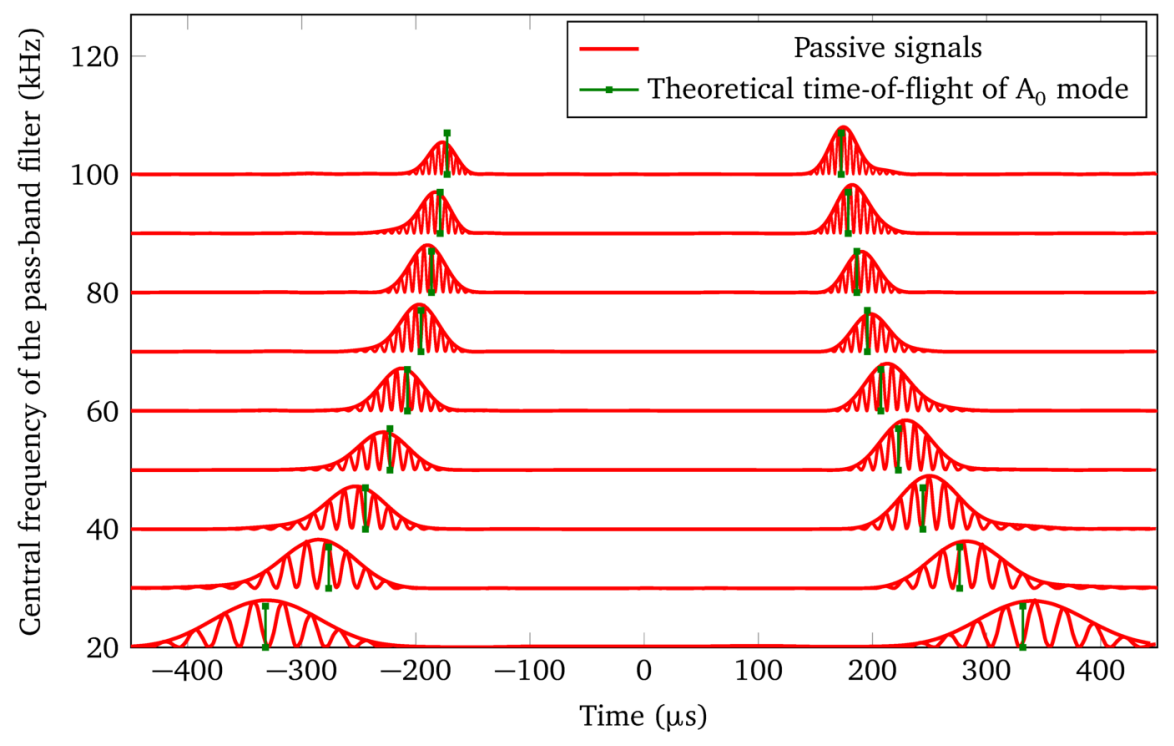

FIG. 3. (Color online) Passive signals similar to the one on Fig. 2 compared to theoretical TOFs of the incident $A_{0}$ mode for several central frequencies. 
On Fig. 3, it should be noted that the $\mathrm{S}_{0}$ mode (fundamental symmetric Lamb mode of the aluminum plate) is missing. When replacing the FBG sensors by $2 \mathrm{PZT}$ transducers of $18 \mathrm{~mm}$ diameter, packets corresponding to the $\mathrm{S}_{0}$ mode are visible at frequencies above $70 \mathrm{kHz}$ (see Fig. 4). The excitation applied by the compressed air jet being almost a normal force only on one surface of the plate, most of the energy is likely to be in the antisymmetric modes, namely $\mathrm{A}_{0}$ for those frequencies. But small amplitude $\mathrm{S}_{0}$ mode is propagating in the plate, as confirmed by PZT measurement.

A different spatial filtering by these two sensors (or "Lamb wave tuning curves", as described by Giurgiutiu (Giurgiutiu , 2005)) due to their different length can explain the fact that FBG sensors did not detected the small amplitude $\mathrm{S}_{0}$ mode in these experiments. The limited length of the FBG sensors (2 mm) makes it a wideband sensor at such low frequencies / long wavelengths. On the contrary, the rejection frequency (i.e. the frequency at which sensitivity is minimal) for $\mathrm{A}_{0}$ mode is $80 \mathrm{kHz}$ for a $18 \mathrm{~mm}$ diameter PZT on a $2 \mathrm{~mm}$ thick aluminum plate, explaining the detection of $\mathrm{S}_{0}$ mode around this frequency. Further investigations should be performed in order to properly characterize spatial filtering characteristics of FBG sensors and to determine configurations where FBG sensors are able to detect $S_{0}$ mode extracted from ambient elastic noise.

\section{B. Cross-correlation of mixed PZT and FBG measurements}

In order to confirm the results of the previous section, and especially demonstrate the good reconstruction of the Green's function thanks to the cross-correlation, active and passive signals between a PZT transducer and an FBG sensor are presented in this section.

In order to obtain active signals, one PZT and one FBG spaced $50 \mathrm{~cm}$ apart from each other were used to respectively emit a guided wave - 5 cycles Hann-windowed $40 \mathrm{kHz}$ toneburst - and receive it after its propagation in the plate. As for the passive reconstruction, the ambient noise was recorded simultaneously with the PZT and the FBG sensor. Then, the cross-correlation was computed and the spectral whitening and equalization to the active signal power spectral density (PSD) - see Eq. 4 - were performed on the cross-correlation to permit the comparison. The obtained active and passive signals are plotted on Fig. 5. Those signals are very similar. The slight differences can be interpreted by the fact that the equipartition hypothesis (see section II A) is not strictly respected by our manual spraying of compressed air jet in order to generate ambient noise field. Therefore Green's function reconstruction is not perfect.

The same experiment is conducted with several distances between sensors, the distance varying between $20 \mathrm{~cm}$ and $60 \mathrm{~cm}$ with a step of $10 \mathrm{~cm}$ (see Fig. 6). This last experiment was conducted to observe the good agreement of the theoretical TOF with both the active and passive data. On Fig. 6 only envelopes are plotted for the sake of clarity. Again, the passive reconstructions are of excellent quality, indicating a very good convergence of ambient noise cross-correlation towards Green's function.

\section{CONCLUSIONS}

It has been demonstrated here that fiber Bragg grating (FBG) sensors on optical fibers can be used in combination with a passive ambient noise cross-correlation technique to retrieve the Green's function between two sensors. The experiments have been performed at ultrasonic frequencies $(20-100 \mathrm{kHz})$ typically used in guided waves based SHM systems.

These results are very promising in order to combine the advantage of guided waves in terms of diagnosis quality with low intrusiveness, environmental immunity and multiplexing capabilities of FBG sensors. This allows to imagine an SHM system made of a network of only FBG sensors embedded in the structure. An adaptation of guided elastic waves imaging algorithms to the directionality behavior of these sensors will have to be performed to demonstrate the effectiveness of this new approach in practical applications.

Future investigations will therefore be devoted to characterize properly FBG as passive sensors of guided elastic waves. In particular, the analysis of FBG directionality's effect on the response will be studied, as well as the capability of these sensors to detect passively not only $\mathrm{A}_{0}$ mode but also $\mathrm{S}_{0}$ mode.

(2013). "Guidelines for Implementation of Structural Health Monitoring on Fixed Wing Aircraft", SAE International.

$\mathrm{Yu}$, L. and Giurgiutiu, V. (2008). "In situ 2-D piezoelectric wafer active sensors arrays for guided wave damage detection", Ultrasonics 48, 117-134.

Sabra, K.G., Winkel, E.S., Bourgoyne, D.A., Elbing, B.R., Ceccio, S.L., Perlin, M., and Dowling, D.R. (2007). "Using cross correlations of turbulent flow-induced ambient vibrations to estimate the structural impulse response. Application to structural health monitoring", Journal of the Acoustical Society of America 121(4), 1987-1995.

Lobkis, O.I., and Weaver, R.L. (2001). "On the emergence of the Green's function in the correlations of a diffuse field", Journal of the Acoustical Society of America 6, 3011-3017.

Sabra, K., Srivastava, A., Lanza di Scalea, F., Bartoli, I., Rizzo, P., and Conti, S. (2008). "Structural health monitoring by extraction of coherent guided waves from diffuse fields", Journal of the Acoustical Society of America 132, 8-13.

Chehami, L., Moulin, E., de Rosny, J., Prada, C., Bou Matar, O., Benmeddour, F., and Assaad, J. (2014). "Detection and localization of a defect in a reverberant plate using acoustic field correlation", Journal of Applied Physics 115, 104901.

Betz, D.C., Thursby, G., Culshaw, B., and Staszewski, W.J. (2003). "Acousto-ultrasonic sensing using fiber Bragg gratings", Smart Materials and Structures 12, 122-128.

Takeda, N., Okabe, Y., Kuwahara, J., Kojima, S., and Ogisu, T. (2005). "Development of smart composite structures with smalldiameter fiber Bragg grating sensors for damage detection: Quantitative evaluation of delamination length in CFRP laminates using Lamb wave sensing", Composites Science and Technology 65(15), 2575-2587.

Sotoudeh, V., Black, R.J., Moslehi, B., and Qiao, P. (2014). "Lamb wave-based damage detection of composite shells using highspeed fiber-optic sensing", Smart Sensor Phenomena, Technology, Networks, and Systems Integration, Proc. of SPIE. 


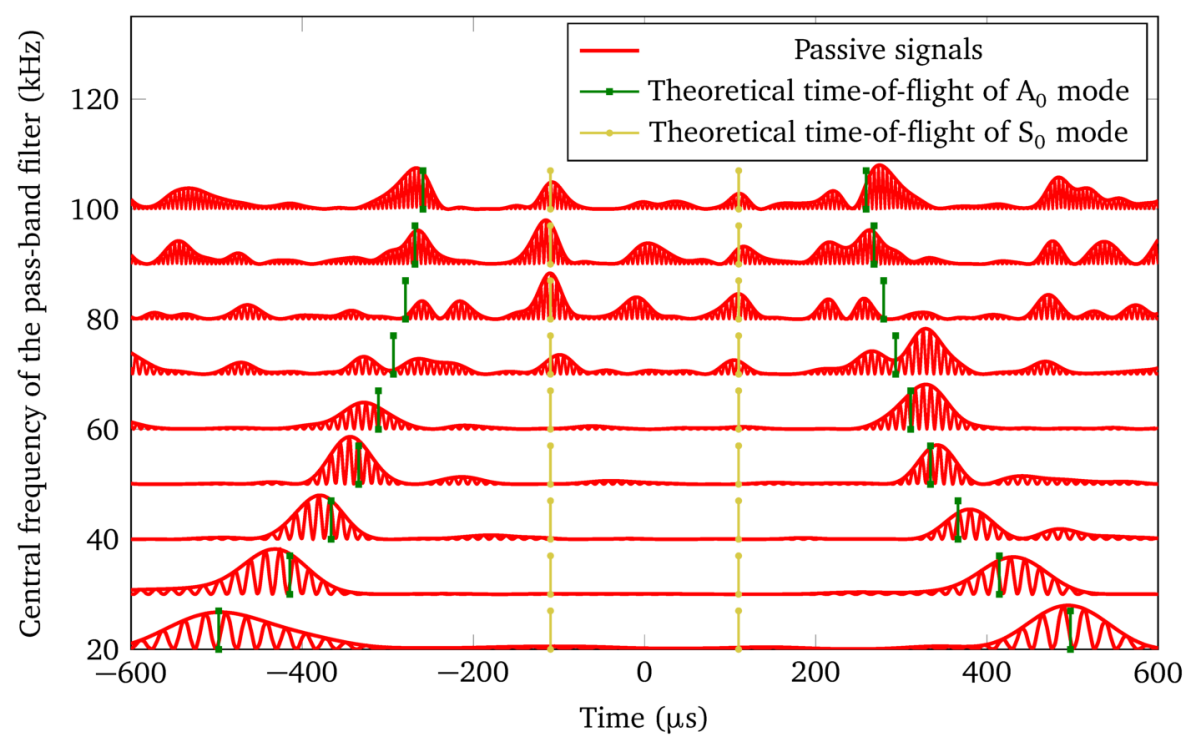

FIG. 4. (Color online) Passive signals between two PZT transducers compared to theoretical TOFs for several central frequencies. $\mathrm{S}_{0}$ mode is visible one this Figure.

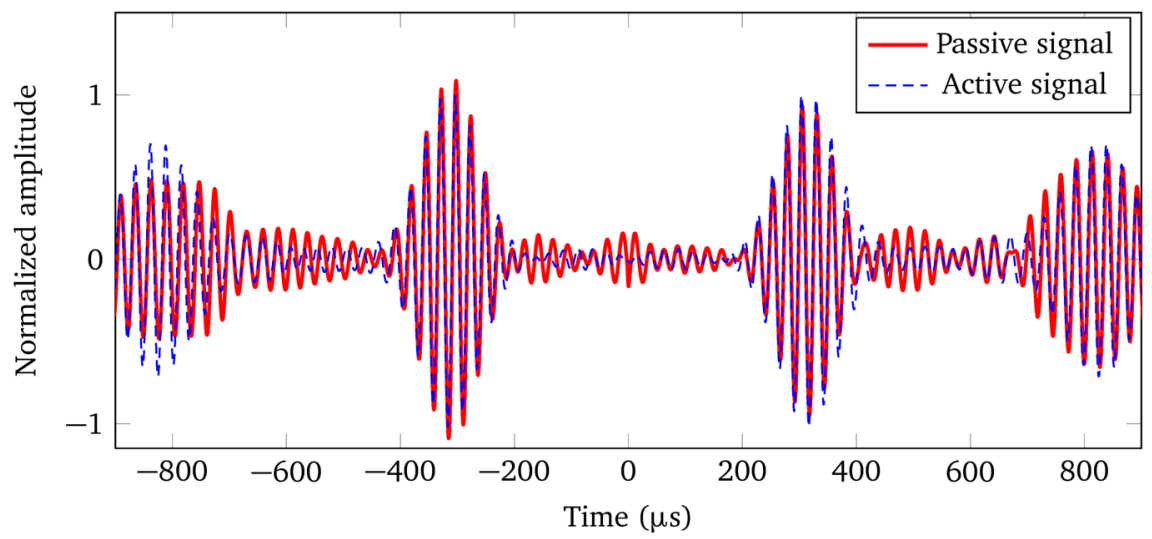

FIG. 5. (Color online) Active and passive signals comparison after PSDs equalization. 
Larose, E., Roux, P., and Campillo, M. (2007). "Reconstruction of Rayleigh-Lamb dispersion spectrum based on noise obtained from an air-jet forcing", Journal of the Acoustical Society of America 122, 3437-3444.

Weaver, R.L., and Lobkis, O.I. (2001). "Ultrasonics without a Source: Thermal Fluctuation Correlations at MHz Frequencies", Physical Review Letters 87(13), 134301.

Derode, A., Larose, E., Tanter, M., de Rosny, J., Tourin, A., Campillo, M., and Fink, M. (2003). "Recovering the Green's function from field-field correlations in an open scattering medium (L)", Journal of the Acoustical Society of America 113, 2973-2976.

Derode, A., Larose, E., Campillo, M., and Fink, M. (2003). "How to estimate the Green's function of a heterogeneous medium between two passive sensors? Application to acoustic waves", Applied Physics Letters 83(15), 3054-3056.

Larose, E., Derode, A., Campillo, M., and Fink, M. (2004). "Imaging from one-bit correlations of wideband diffuse wave fields", Journal of Applied Physics 95, 8393-8399.

Davy, M., de Rosny, J., and Besnier, P. (2016). "Green's Function Retrieval with Absorbing Probes in Reverberating Cavities", Phys. Rev. Lett. 116, 213902.

Larose, E., and Hall, S. (2009). "Monitoring stress related velocity variation in concrete with a $2 \times 10-5$ relative resolution using diffuse ultrasound", The Journal of the Acoustical Society of America 125(4), 1853-1856.

Duroux, A., Sabra, K.G., Ayers, J., and Ruzzene, M. (2010). "Using cross-correlations of elastic diffuse fields for attenuation tomography of structural damage", Journal of the Acoustical Society of America 127, 3311-3314.

Duroux, A., Sabra, K.G., Ayers, J., and Ruzzene, M. (2010). "Extracting guided waves from cross-correlations of elastic diffuse fields: Applications to remote structural health monitoring", Journal of the Acoustical Society of America 127, 204-215.

Sabra, K.G., and Huston, S. (2011). "Passive structural health monitoring of a high-speed naval ship from ambient vibrations", Journal of the Acoustical Society of America 129, 2991-2999.

Roux, P., Kuperman, W.A., Colosi, J.A., Cornuelle, B.D., Dushaw, B.D., Dzieciuch, M.A., Howe, B.M., Mercer, J.A., Munk, W., Spindel, R.C., and Worcester, P.F. (2004). "Extracting coherent wave fronts from acoustic ambient noise in the ocean", The Journal of the Acoustical Society of America 116(4), 1995-2003.

Sabra, K.G., Roux, P., and Kuperman, W.A. (2005). "Emergence rate of the time-domain Green's function from the ambient noise cross-correlation function", The Journal of the Acoustical Society of America 118(6), 3524-3531.

Zang, X., Brown, M.G., and Godin, O.A. (2015). "Waveform modeling and inversion of ambient noise cross-correlation functions in a coastal ocean environment", The Journal of the Acoustical Society of America 138(3), 1325-1333.

Duvall, T.L., Jefferies, S.M., Harvey, J.W., and Pomerantz, M.A. (1993). "Time-distance helioseismology", The Journal of the Acoustical Society of America.

Gizon, L., and Birch, A.C. (2004). "Time-distance helioseismology: noise estimation", The Astrophysical Journal 614(1), 472.

Zorgani, A., Souchon, R., Dinh, A.-H., Chapelon, J.-Y., Ménager, J.-M., Lounis, S., Rouvière, O., and Catheline, S. (2015). "Brain palpation from physiological vibrations using MRI", Proceedings of the National Academy of Sciences 112(42), 12917-12921.

Sabra, K.G., Conti, S., Roux, P., and Kuperman, W.A. (2007). "Passive in vivo elastography from skeletal muscle noise", Applied physics letters 90(19), 194101.

Campillo, M., and Paul, A. (2003). "Long-Range Correlations in the Diffuse Seismic Coda", Science 299, 547-549.

Shapiro, N.M., Campillo, M., Stehly, L., and Ritzwoller, M.H. (2005). "High-resolution surface-wave tomography from ambient seismic noise", Science 307(5715), 1615-1618.

Shapiro, N.M., and Campillo, M. (2004). "Emergence of broadband Rayleigh waves from correlations of the ambient seismic noise", Geophysical Research Letters 31(7).

Sabra, K.G., Gerstoft, P., Roux, P., Kuperman, W.A., and Fehler, M.C. (2005). "Surface wave tomography from microseisms in Southern California", Geophysical Research Letters 32(14).

Snieder, R., and Wapenaar, K. (2010). "Imaging with ambient noise", Physics Today 63, 44-49.
Zeng, X., Lancelle, C., Thurber, C., Fratta, D., Wang, H., Lord, N. Chalari, A., and Clarke, A. (2017). "Properties of noise crosscorrelation functions obtained from a distributed acoustic sensing array at Garner Valley, California", Bulletin of the Seismological Society of America 107(2), 603-610.

Giurgiutiu, V. (2005). "Tuned Lamb Wave Excitation and Detection with Piezoelectric Wafer Active Sensors for Structural Health Monitoring", Journal of Intelligent Material Systems and Structures 16(4), 291-305. 


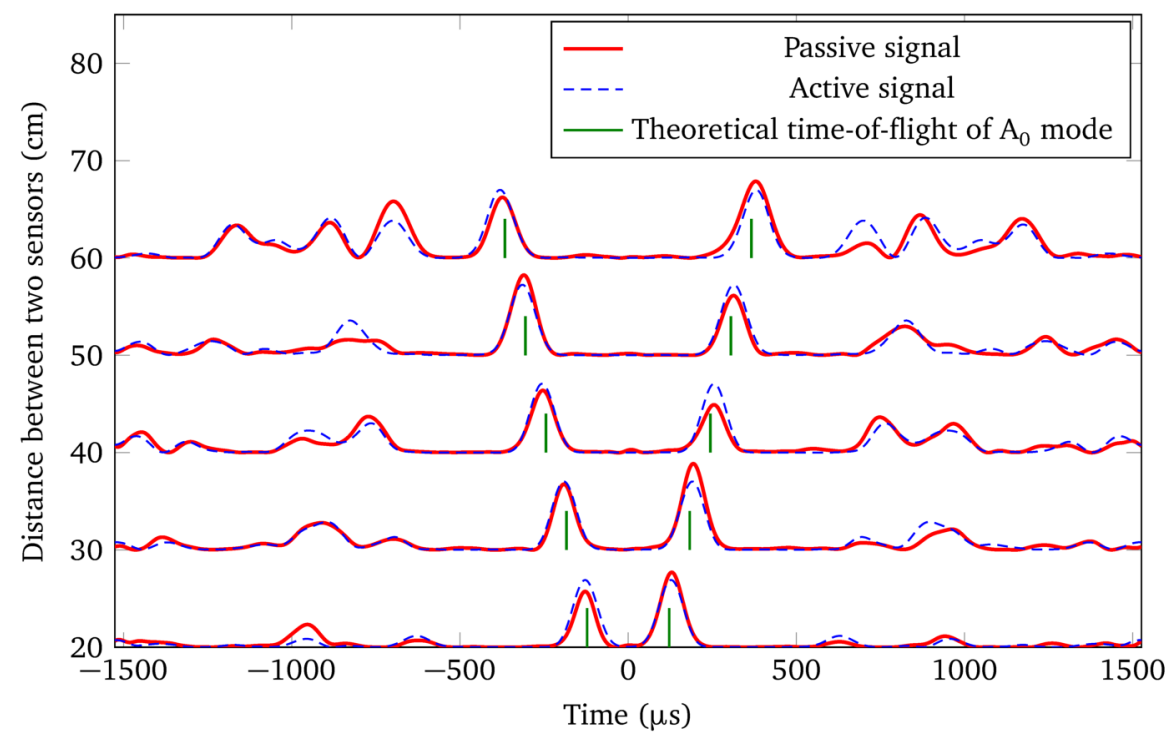

FIG. 6. (Color online) Active signal and passive signal envelope comparison after equalization of the PSDs for different distances between sensors. 